\title{
Combining Targeted Metabolite Analyses and Transcriptomics to Reveal Specific Chemical Composition and Associated Genes in Resistant Soybean Infected with Soybean Cyst Nematode
}

\section{Xue Shi}

Chinese Academy of Agricultural Sciences Institute of Plant Protection

\section{Qiansi Chen}

Zhengzhou Tobacco Research Institute

Shiming Liu

Chinese Academy of Agricultural Sciences Institute of Plant Protection

Jiajun Wang

Heilongjiang Academy of Agricultural Sciences

\section{Deliang Peng}

Chinese Academy of Agricultural Sciences Institute of Plant Protection

\section{Lingan Kong ( $\sim$ konglingan@caas.cn )}

Chinese Academy of Agricultural Sciences Institute of Plant Protection

\section{Research article}

Keywords: resistant and susceptible soybeans, soybean cyst nematode, metabolomic analyses, transcriptomics, combination analyses

Posted Date: October 5th, 2020

DOI: https://doi.org/10.21203/rs.3.rs-73506/v1

License: (a) (i) This work is licensed under a Creative Commons Attribution 4.0 International License. Read Full License 


\section{Abstract}

Background: Soybean cyst nematode Heterodera glycines is one of the most devastating pathogens on soybean and causes severe annual yield loss worldwide. Different soybean varieties exhibit different responses to soybean cyst nematode infection at various levels, such as genomic, transcriptional, proteomic, and metabolomic levels. However, there were not yet any reports on the differential responses of resistant and susceptible soybeans infected with soybean cyst nematode by combining the metabolomic analyses and transcriptomics.

Results: In this study, a highly-resistant variety PI 437654 and three susceptible varieties Williams 82, Zhonghuang 13 and Hefeng 47 were used as the test materials to clarify the differences in metabolites and transcriptomics between resistant and susceptible soybeans before and after SCN infection. A local metabolite-calibrated database was used to identify potential differential metabolites, and the differences of metabolites and metabolic pathways were compared between the resistant and susceptible soybean varieties after inoculation with SCN. Totally, 37 differential metabolites and 20 KEGG metabolic pathways were identified, which were divided into three categories including the metabolites/pathways overlapped among resistant and susceptible soybeans, specific in susceptible or resistant soybeans, respectively. Twelve differential metabolites were found to be involved in predicted KEGG metabolite pathways. Moreover, 14 specifically differential metabolites such as significantly up-regulated nicotine and downregulated D-aspartic acid, and their involved KEGG pathways such as tropane, piperidine and pyridine alkaloid biosynthesis, alanine, aspartate and glutamate metabolisms, sphingolipid metabolism and arginine biosynthesis were significantly changed and abundantly enriched in the resistant soybean, and likely played pivotal roles in defensing against SCN infection. Three key metabolites including Nacetyltranexamic acid, nicotine, and D, L-typotophan, which were found to be significantly up-regulated in the resistant soybean PI437654 infected by SCN, classified into two types and used for combination analyses with the transcriptomic expression profiling. Associated genes were predicted, and suggested their likely biological processes, cellular component, molecular function and involved pathways.

Conclusions: Our results not only found out the potential novel metabolites and associated genes in the resistant response of soybean to soybean cyst nematode, but also provided new insights into the interactions between soybean and soybean cyst nematode.

\section{Background}

Soybean cyst nematode (SCN), Heterodera glycines, is one of the most devastating pathogens on soybean and causes a large quantity of annual yield loss worldwide [17, 40,49]. SCN is a typical obligate endoparasitic nematode, its life cycle has three stages: egg, juvenile and adult. Juvenile stage is a nofeeding stage of infection. The juveniles pierce the soybean root with a spear-like feeding structure called stylet to penetrate the soybean root $[21,49]$. They invade plant roots and then migrate to vascular bundles, where they establish a complex feeding site called syncytium [30,44]. The formation and maintenance of the syncytium lead to dramatic changes in internal root structure, affect plant root 
system, and cause plant damages. Meanwhile, soybean roots produce many defense-related compounds such as reactive oxygen species and activate hormone signaling pathways in response to nematode infection [27, 31], and the metabolites undergo tremendous changes in the soybean roots upon infection of SCN.

Recently, the use of "omics" technology to obtain biological data has been surged, the most commonly used omics are genomics, transcriptomics, proteomics, and metabolomics [53]. There are numerous research reports about soybean-SCN interactions by genomic and transcriptomic analyses. The genomes of SCN and many soybean varieties were uncovered as the solid foundations to further investigate their biology, characteristics and their potential interactions [38, 43, 61]. Many soybean resistant candidate loci and/or genes were identified based on the genome-wide association study (GWAS) [24, 59, 64]. Moreover, many studies on the expression profiling of different plant tissues, such as the whole root samples and the syncytium structure were carried out in susceptible and resistant soybean varieties infected with SCN, and a lot of differential expression genes and related KEGG pathways were predicted [34, 35, 45]. However, there are few studies on soybean-SCN interactions by proteomic and metabolomic analyses.

Metabolomics is used to characterize the metabolic patterns and further study the functions and significance of the key differential metabolites, which play important roles in biological and medical fields[42]. Plant metabolomics has gained cumulative attentions, and became a mature method for studying plant responses to both biotic and abiotic stresses. Therefore, plant metabolomics is considered as an indispensable tool in the study of plant-pathogen interaction [12]. Plants respond to pathogen infection by producing the defense metabolites, especially, the plant secondary metabolites play important roles in plant host's resistance [33]. Metabolomics will provide technical support for the identification of plant metabolites which are potential sources of new compounds for nematode control [9]. Research on plant nematodes has become more and more abundant, especially on root-knot nematodes. Eloh et al. (2016) performed metabolomic analysis of root-knot nematodes treated with maleimide, and proved that maleimide could be used as a new potential nematicide [22]. In addition, they studied the levels of metabolites in tomato plants after root-knot nematode infection, and found that tomato roots altered the biochemical pathways in response to root-knot nematode infection [22]. Recently, Borges et al. (2018) investigated the metabolic profiles of both resistant and susceptible watermelon varieties against root-knot nematode infection by using nuclear magnetic resonance (NMR) and principal component analysis (PCA) [9]. However, there was few reports on SCN-soybean interactions. Differential proteins and metabolites between the resistant and susceptible soybean roots were identified with the traditional approaches more than ten years ago [1]. Recently, Kang et al. (2018) studied the effect of Bacillus simplex strain Sneb545 on soybean secondary metabolites under SCN infection by combining the advanced and high-throughput approaches of transcriptomic and metabolomic analyses, and found that Sneb545-treated soybeans showed a higher concentration of various nematicidal metabolites [32].

To date, there were no reports on the difference of metabolic pathways between resistant and susceptible soybeans with infection of SCN. In this study, a SCN-resistant soybean variety PI $437654[3,4]$, and three 
SCN-susceptible soybean varieties Williams 82 (WM82), Zhonghuang 13 (ZH13) and Hefeng 47 (HF47) were selected as the research materials, and LC/MS full-scan detection technology was used to investigate the differences of metabolites and metabolic pathways between the resistant and susceptible soybean varieties after inoculation with $\mathrm{SCN}$. The metabolites overlapped among resistant and susceptible soybeans, specifically differential metabolites in resistant- or susceptible-soybeans and their potential KEGG metabolic pathways were identified. Moreover, the genes associated with the significantly up-regulated metabolites in the resistant soybean, which mainly included the resistant soybean PI437654-specific up-regulated and the overlapping metabolite with significantly up-regulated but simultaneously down-regulated metabolites, were predicted. The results not only found out potential novel metabolites and associated genes in the resistant response of soybean to soybean cyst nematode, but also provided new insights into the interaction between soybean and soybean cyst nematode.

\section{Results}

\section{Assessment of resistance of soybean to SCN}

At $8 \mathrm{dpi}$ of SCN race 4, the average numbers of the second juveniles (J2s) within PI 437654 roots were significantly less than that of ZH13, while it did not show significant difference among PI 437654, WM82 and HF47 (Fig. 1a). However, the average numbers of the third juveniles (J3s) in PI 437654 were significantly decreased than those in the three other varieties (Fig. 1a; Fig. 1b). At 60 dpi, the average numbers of cysts formed in the soybean PI 437654 were significantly decreased than that in the three other soybeans (WM82, ZH13 and HF47) (Fig. 1a). In addition, the J3s were much slimmer in the PI 437654 roots than in the three other soybeans (Fig. 1b). These results indicated that PI 437654 was a highly SCN-resistant soybean variety, while the other three varieties were the highly SCN-susceptible soybeans.

\section{Differentiation of the metabolites in resistant and susceptible soybeans by the infection of SCN}

To investigate the differential root metabolites between the resistant and susceptible soybeans, the root samples of the resistant soybean PI 437654 and the three susceptible soybeans (WM82, ZH13 and HF47) inoculated with SCN race 4 ('_SCN') at $8 \mathrm{dpi}$, as well as their corresponding controls ('_0'), were collected and subjected to metabolomic analyses (PCA and PLS-DA analyses). In the PCA score chart of resistant soybean, all the PI 437654_SCN replicated samples were clustered together, and similarly, all the PI 437654_0 replicates were also clustered together (Fig. 2a), suggesting that the samples had sound repeatability. However, the PI 437654_SCN samples were clearly separated from the PI437654_0 samples (Fig. 2a), illustrating the significant induction of the changes of metabolites in the resistant soybean PI 437654 by the inoculation of SCN race 4 and the solid stability in the whole measurement process. The PLS-DA analyses results were similar to those of the PCA analyses, the samples of PI 437654_SCN were also clearly separated from those of PI437654_0 (Fig. 2b). The PCA and PLS-DA analyses results showed 
that all the three susceptible soybean lines WM82_SCN, ZH13_SCN and HF47_SCN were dramatically separated from each WM82_0, ZH13_0, and HF47_0 pairwise (Fig. 2a; Fig. 2b), respectively, which indicated that the inoculation of SCN also caused obvious changes of metabolites of the susceptible soybean roots. Furthermore, according to the cumulative interpretation rate of model in the resistant and susceptible soybeans (Fig. 2c; Table S1), both $\mathrm{R}^{2}$ and $\mathrm{Q}^{2}$ values were closer to 1 , clearly explaining that the predictive power and model quality of the two groups of models were good and suitable for the subsequent experiments. All the results indicated that the SCN inoculation caused significant metabolic changes in both resistant and susceptible soybean lines.

\section{Identification of differential metabolites in the resistant and susceptible soybeans}

At 8 dpi of SCN, 19 significantly differential metabolites were screened out in the resistant soybean PI 437654_SCN vs PI 437654_0 pairwise, among which seven metabolites were up-regulated, and 12 metabolites were down-regulated (Table 1). In the susceptible soybean WM82_SCN vs WM82_0 pairwise, 17 obviously differential metabolites were identified, including three up-regulated and 14 down-regulated metabolites (Table 1). Similarly, there were 12 dramatically differential metabolites in ZH13_SCN vs ZH13_0 pairwise, all of them were down-regulated (Table 1). There were 17 dramatically differential metabolites in HF47_SCN vs HF47_0 pairwise, including three up-regulated metabolites and 14 downregulated metabolites (Table 1). The differential metabolites among the four soybean lines had different profiles, which were mainly divided into following three categories. 
Table 1

Differential metabolites in the roots of the resistant and susceptible soybeans

\begin{tabular}{|c|c|c|c|c|}
\hline Metabolites & PI 437654* & WM82 & ZH13 & HF47 \\
\hline Linolenic acid & -1.604 & -1.254 & -1.741 & -1.172 \\
\hline D-leucine & -0.759 & 0.322 & -2.313 & -1.289 \\
\hline 16-Hydroxyhexadecanoic acid & -1.100 & -0.537 & -1.138 & 0.685 \\
\hline DL-Typotophan & 1.300 & -0.544 & -0.892 & -0.556 \\
\hline D-aspartic acid & -8.796 & / & / & / \\
\hline Linoleic acid & -6.199 & / & I & / \\
\hline N-palmitoyl alanine & -3.060 & / & / & / \\
\hline Cycloleucine & -1.632 & / & / & / \\
\hline D,L-2,4-Diaminobutyric acid & -1.307 & / & / & / \\
\hline Phytosphingosine & -0.987 & / & / & / \\
\hline 10-oxo-nonadecanoic acid & -0.885 & I & I & I \\
\hline N-Acetyltranexamic acid & 3.688 & / & / & / \\
\hline Nicotine & 2.543 & I & / & / \\
\hline L-Arginine & 0.893 & / & / & / \\
\hline Pipecolinic acid & -2.026 & -1.591 & -1.390 & / \\
\hline Gallocatechin gallate & -1.688 & -1.573 & / & / \\
\hline 4-Methylquinoline & 1.110 & / & / & -0.584 \\
\hline Nicotyrine & 1.096 & / & / & -0.563 \\
\hline L-trans-4-hydroxy-L-proline & 1.047 & / & / & -0.556 \\
\hline 2-0xo-4-methylthiobutanoic acid & / & -0.852 & -1.311 & -0.474 \\
\hline 4-Hydroxycoumarin & / & -0.933 & -1.293 & -0.430 \\
\hline $\mathrm{N}$-Cyclohexanecarbonylpentadecylamine & / & -2.816 & -4.143 & / \\
\hline $\mathrm{PC}(0-14: 0 / 2: 0)$ & / & -2.613 & / & 1.522 \\
\hline Isoliquiritigenin & / & -2.000 & -3.391 & / \\
\hline Rhamnazin & / & -1.037 & / & / \\
\hline \multicolumn{5}{|c|}{$\begin{array}{l}\text { Note: ' } * \text { ' represents the } \log _{2}(\mathrm{FC}) \text { value which was the ratio of the average expression of metabolites } \\
\text { in the sample batch. Positive value represents up-regulated, while negative value represents down- } \\
\text { regulated. "/' represents that soybean varieties did not contain such metabolites. }\end{array}$} \\
\hline
\end{tabular}




\begin{tabular}{|c|c|c|c|c|}
\hline Metabolites & PI 437654* & WM82 & $\mathrm{ZH} 13$ & HF47 \\
\hline Betulinic acid & / & -1.020 & / & / \\
\hline Drimenol & / & -0.923 & -2.296 & / \\
\hline 3-Hydroxy-7-methoxyflavone & / & 2.170 & -7.687 & / \\
\hline Prunetin & / & 1.637 & / & / \\
\hline Baicalein & / & / & -1.560 & / \\
\hline ent-kaur-16-en-19-ol & / & / & / & -5.012 \\
\hline isopimaric acid & I & l & l & -3.091 \\
\hline Communic acid & l & l & l & -2.529 \\
\hline Hydroxy citronellal & / & / & / & -1.470 \\
\hline Hexadecanamide & / & / & / & -1.324 \\
\hline Dichotosinin & / & -2.044 & / & -1.212 \\
\hline trans-Ferulic acid & / & / & / & 3.011 \\
\hline \multicolumn{5}{|c|}{$\begin{array}{l}\text { Note: '*' represents the } \log _{2}(\mathrm{FC}) \text { value which was the ratio of the average expression of metabolites } \\
\text { in the sample batch. Positive value represents up-regulated, while negative value represents down- } \\
\text { regulated. "/' represents that soybean varieties did not contain such metabolites. }\end{array}$} \\
\hline
\end{tabular}

(1) Differential metabolites overlapped among resistant and susceptible soybeans. There were four differential metabolites overlapped (Table 1; Fig. S1), including D-leucine, D, L-typotophan, 16hydroxyhexadecanoic acid and linolenic acid, which belong to amino acids and fatty acids. Overall, linolenic acid, D-leucine and 16-Hydroxyhexadecanoic acid had an overall significantly down-regulated in resistant and three susceptible soybeans. However, D-leucine and 16-hydroxyhexadecanoic acid were obviously up-regulated in WM82_SCN vs WM82_0, and HF47_SCN vs HF47_0, respectively. Differently, D, L-typotophan was simultaneously dramatically down-regulated in the three susceptible soybean pairwise, while significantly up-regulated in the resistant soybean PI 437654_SCN vs PI437654_0 pairwise (Table 1). The results indicated that $\mathrm{D}$, L-typotophan potentially played an important role to defense SCN in the resistant soybean PI 437654.

(2) Differential metabolites specific in the susceptible soybeans. There were 18 significantly differential metabolites specifically presented in the susceptible soybeans but absented in the resistant PI437654_SCN vs PI437654_0. Both 2-oxo-4-methylthiobutanoic acid and 4-hydroxycoumarin were simultaneously overlapped in all the three susceptible soybeans (Table 1; Fig S1), while the other 16 metabolites only existed in one or two of the three susceptible soybeans (Table 1). Except four metabolites including PC (0-14:0/2:0), 3-hydroxy-7-methoxyflavone, prunetin, and trans-ferulic acid were dramatically up-regulated in either WM82 or HF47 pairwise, the other 14 metabolites were obviously down-regulated among the three susceptible soybeans (Table 1). The suppressed differential metabolites 
in the susceptible soybeans were probably linked to the soybean basal defense hijacked by the SCN infection for their establishment and development of the feed sites.

(3) Differential metabolites specific in the resistant soybean. There were 10 uniquely differential metabolites in the resistant soybean PI 437654_SCN vs PI 437654_0 pairwise (Table 1; Fig. S1), including 7 down-regulated and 3 up-regulated metabolites (Table 1). Both D-aspartic acid and linoleic acid were the top two significantly down-regulated metabolites with about 70 -fold more abundance in PI437654_SCN than that in PI 437654_0 (Table 1). N-palmitoyl alanine, cycloleucine and D, L-2,4diaminobutyric acid were all the sequentially differential down-regulated metabolites at about 8-, 3- and 2.5-fold more in PI 437654_SCN than that in PI 437654_0, respectively (Table 1). Among the 3 significantly up-regulated metabolites, $\mathrm{N}$-acetyltranexamic acid and nicotine were the top two upregulated metabolites at about 12.9- and 5.8-fold more in PI 437654_SCN than in PI 437654_0, respectively (Table 1). In addition, 5 obviously differential metabolites including two down-regulated and three up-regulated metabolites in the PI437654_SCN vs PI437654_0 pairwise were identified. However, these five metabolites were all reduced but not existed in all the three susceptible soybeans (Table 1). These results indicated that $\mathrm{N}$-acetyltranexamic acid and nicotine might play potential roles to defense SCN infection in PI 437654, while D-aspartic acid and linoleic acid and N-palmitoyl alanine were likely involved into malfunction in feeding site establishment and maintenance for SCN development in PI 437654.

\section{Metabolic pathways involved by differential metabolites in the resistant and susceptible soybeans}

The metabolic pathways involved by the differential metabolites were analyzed by the KEGG database searching. Totally, 14 metabolic pathways were changed in the resistant soybean PI 437654_SCN vs PI 437654_0, while 9, 9 and 8 metabolic pathways were changed in the susceptible soybean WM82_SCN vs WM82_0, ZH13_SCN vs ZH13_0, HF47_SCN vs HF47_0, respectively (Fig. 3, Table 2), which could also be divided into following three categories. 
Table 2

Analyses of the metabolic pathways involved by the differential metabolites in the resistant and susceptible soybeans

\begin{tabular}{|c|c|c|c|c|c|c|}
\hline Metabolite & KEGG ID & Annotation & PI437654* & WM82 & $\begin{array}{l}\mathrm{ZH} \\
13\end{array}$ & HF47 \\
\hline $\begin{array}{l}16- \\
\text { Hydroxyhexadecanoic } \\
\text { acid }\end{array}$ & ath00073 & $\begin{array}{l}\text { Cutin, suberine and } \\
\text { wax biosynthesis }\end{array}$ & 1.337 & 1.480 & 1.480 & 1.402 \\
\hline \multirow[t]{2}{*}{ Linolenic acid ${ }^{\#}$} & ath00592 & $\begin{array}{l}\text { alpha-Linolenic acid } \\
\text { metabolism }\end{array}$ & 1.130 & 1.272 & 1.272 & 1.195 \\
\hline & ath01040 & $\begin{array}{l}\text { Biosynthesis of } \\
\text { unsaturated fatty } \\
\text { acids }\end{array}$ & 0.914 & 1.052 & 1.052 & 0.977 \\
\hline \multirow[t]{3}{*}{$\begin{array}{l}\text { 2-0xo-4- } \\
\text { methylthiobutanoic } \\
\text { acid } \#\end{array}$} & ath00270 & $\begin{array}{l}\text { Cysteine and } \\
\text { methionine } \\
\text { metabolism }\end{array}$ & l & 1.134 & 1.133 & 1.058 \\
\hline & ath00966 & $\begin{array}{l}\text { Glucosinolate } \\
\text { biosynthesis }\end{array}$ & l & 1.036 & 1.036 & 0.961 \\
\hline & ath01210 & $\begin{array}{l}\text { 2-0xocarboxylic acid } \\
\text { metabolism }\end{array}$ & l & 0.808 & 0.808 & 0.735 \\
\hline Isoliquiritigenin & ath00941 & $\begin{array}{l}\text { Flavonoid } \\
\text { biosynthesis }\end{array}$ & l & 1.052 & 1.052 & I \\
\hline isopimaric acid & \multirow{2}{*}{$\begin{array}{l}\text { ath00904 } \\
\text { \& }\end{array}$} & \multirow{2}{*}{$\begin{array}{l}\text { Diterpenoid } \\
\text { biosynthesis }\end{array}$} & \multirow[t]{2}{*}{ / } & \multirow[t]{2}{*}{ / } & \multirow[t]{2}{*}{ / } & \multirow[t]{2}{*}{1.906} \\
\hline ent-kaur-16-en-19-ol & & & & & & \\
\hline trans-Ferulic acid & ath00940 & $\begin{array}{l}\text { Phenylpropanoid } \\
\text { biosynthesis }\end{array}$ & / & / & / & 1.025 \\
\hline Phytosphingosine & ath00600 & $\begin{array}{l}\text { Sphingolipid } \\
\text { metabolism }\end{array}$ & 1.370 & / & l & / \\
\hline D-aspartic acid & ath00250 & $\begin{array}{l}\text { Alanine, aspartate } \\
\text { and glutamate } \\
\text { metabolism }\end{array}$ & 1.321 & l & l & I \\
\hline \multirow[t]{3}{*}{ L-Arginine $\#$} & ath00220 & $\begin{array}{l}\text { Arginine } \\
\text { biosynthesis }\end{array}$ & 1.405 & / & / & / \\
\hline & ath00261 & $\begin{array}{l}\text { Monobactam } \\
\text { biosynthesis }\end{array}$ & 1.181 & / & l & / \\
\hline & ath00970 & $\begin{array}{l}\text { Aminoacyl-tRNA } \\
\text { biosynthesis }\end{array}$ & 1.060 & / & / & / \\
\hline
\end{tabular}

Note: ' $*$ ' represents -log10 ( $p$-value) value, while the $p$-value represents the $p$ value of the metabolic pathways. The smaller the $p$ value is, the greater the value of -log (p-value) is. ' $\#$ ' means that the same metabolite appeared in different metabolic pathways. ' $\&$ ' means that different metabolites appeared in the same metabolic pathway. 


\begin{tabular}{|c|c|c|c|c|c|c|}
\hline Metabolite & KEGG ID & Annotation & PI437654* & WM82 & $\begin{array}{l}\mathrm{ZH} \\
13\end{array}$ & HF47 \\
\hline & ath00330 & $\begin{array}{l}\text { Arginine and proline } \\
\text { metabolism }\end{array}$ & 0.893 & / & l & / \\
\hline & ath00999 & $\begin{array}{l}\text { Biosynthesis of } \\
\text { secondary } \\
\text { metabolites - } \\
\text { unclassified }\end{array}$ & 0.792 & / & / & / \\
\hline & ath02010 & $A B C$ transporters & 0.700 & / & / & / \\
\hline & ath01230 & $\begin{array}{l}\text { Biosynthesis of } \\
\text { amino acids }\end{array}$ & 0.693 & / & / & / \\
\hline Nicotine & \multirow{2}{*}{$\begin{array}{l}\text { ath00960 } \\
\&\end{array}$} & \multirow{2}{*}{$\begin{array}{l}\text { Tropane, piperidine } \\
\text { and pyridine alkaloid } \\
\text { biosynthesis }\end{array}$} & \multirow[t]{2}{*}{2.253} & / & / & / \\
\hline \multirow[t]{2}{*}{ Pipecolinic acid \# } & & & & 1.088 & 1.088 & / \\
\hline & ath00310 & Lysine degradation & 1.037 & 1.177 & 1.177 & / \\
\hline \multicolumn{7}{|c|}{$\begin{array}{l}\text { Note: ' } * \text { ' represents -log10 ( } p \text {-value) value, while the } p \text {-value represents the } p \text { value of the metabolic } \\
\text { pathways. The smaller the } p \text { value is, the greater the value of -log (p-value) is. '\#' means that the same } \\
\text { metabolite appeared in different metabolic pathways. ' } \& \text { ' means that different metabolites appeared } \\
\text { in the same metabolic pathway. }\end{array}$} \\
\hline
\end{tabular}

(1) KEGG metabolic pathways overlapped among the resistant and susceptible soybeans. Three KEGG metabolic pathways including cutin, suberine and wax biosynthesis (ath00073), alpha-Linolenic acid metabolism (ath00592), and biosynthesis of unsaturated fatty acids (ath01040) were associated with the differential metabolites 16-hydroxyhexadecanoic acid and linolenic acid (Table 2) and overlapped among all the resistant and susceptible soybeans. Moreover, the cutin, suberine and wax biosynthesis KEGG metabolic pathway (ath00073) was obviously activated in all the resistant soybean PI 437654 and the three susceptible soybeans infected with SCN (Fig. 3, Table 2), which indicated that this KEGG metabolic pathway was likely involved in the basal defense response of soybean varieties against SCN infection.

(2) KEGG metabolic pathways specific in the susceptible soybeans. Three KEGG metabolic pathways, including cysteine and methionine metabolism (ath00270), glucosinolate biosynthesis (ath00966) and 2Oxocarboxylic acid metabolism (ath01210) associated with the differential metabolite 2-oxo-4methylthiobutanoic acid were simultaneously overlapping among all the three susceptible soybeans (Table 2). However, these three KEGG metabolic pathways had not significantly changed (Fig. 3; Table 2).

(3) KEGG metabolic pathways specific in the resistant soybeans. Nine resistant specific KEGG metabolic pathways related with three differential metabolites L-arginine, phytosphingosine and D-aspartic acid were identified, which included sphingolipid metabolism (ath00600), alanine, aspartate and glutamate metabolism (ath00250), arginine biosynthesis (ath00220), monobactam biosynthesis (ath00261), aminoacyl-tRNA biosynthesis (ath00970), arginine and proline metabolism (ath00330), biosynthesis of secondary metabolites-unclassified (ath00999), ABC transporters (ath02010), and biosynthesis of amino acids (ath01230) (Table 2). Although the tropane, piperidine and pyridine alkaloid biosynthesis KEGG 
metabolic pathway (ath00960) was the most obviously changed in the PI 437654 (Fig. 3), it was not included in the resistant specific type, because this KEGG metabolic pathway was also present in both WM82 and ZH13 but absent in HF47. Among these nine KEGG pathways, sphingolipid metabolism (ath00600), alanine, aspartate and glutamate metabolism (ath00250) and arginine biosynthesis (ath00220) were all dramatically changed (Fig. 3; Table 2), suggesting their prevailing roles against SCN infection in the resistant soybean PI 437654.

\section{Associated genes involved in the up-regulated metabolites of the resistant soybean variety}

In order to predict the associated genes involved in the up-regulated metabolites of the resistant soybean variety PI437654, the transcriptome of the resistant soybean PI437654 and the three susceptible soybean WM82, ZH13 and HF47, as well as their corresponding controls, were sequenced, and these transcriptome data were combined with the metabolomic data. The comparative transcriptome analyses identified 15,835 differential expressed genes (DEGs) (6,922_UP VS 8,913_DOWN), 12,225 DEGs (6,001_UP VS 6,224_DOWN), 18,362 DEGs (9,589_UP VS 8,773_DOWN) and 19,528 DEGs (8,944_UP VS 10,584_DOWN) in the resistant soybean PI437654 and the three susceptible soybean WM82, ZH13 and HF47, respectively, infected by SCN (Fig S2). As mentioned above, three key metabolites including Nacetyltranexamic acid, nicotine, and D, L-typotophan, which were significantly up-regulated in the resistant soybean PI437654 infected by SCN, were classified into two types.

One type is the resistant soybean PI437654-specific significantly up-regulated metabolites containing Nacetyltranexamic acid and nicotine. The results of combination analyses showed that 14 potential associated genes (10 positive and 4 negative correlations) were simultaneously linked to both $\mathrm{N}$ acetyltranexamic acid and nicotin, while 68 (52 positive and 16 negative correlations) and 54 (21 positive and 33 negative correlations) associated genes were specific linked to individual nicotin and $\mathrm{N}$ acetyltranexamic acid, respectively (Fig. 4; Table S2). These associated genes were subject to GO analyses. The most abundant GO terms in the biological processes category were "translation", followed by "cytoplasmic translation" and "rRNA modification" (Fig. 5; Table S3). Regarding the cellular component category, the most abundant GO terms were "ribosome", "cytosolic ribosome", "cytosolic small ribosomal subunit", cytosolic large ribosomal subunit" and "nucleolus" (Fig. 5; Table S3). Concerning the molecular function category, the most abundant GO terms were "structural constituent of ribosome", "mRNA binding", "rRNA binding", "RNA binding" and "inorganic cation transmembrane transporter activity" (Fig. 5; Table S3). Correspondingly, these associated genes were enriched in KEGG pathways, which involving the largest number of unigenes were "Ribosome", followed by "RNA degradation", "Ribosome biogenesis in eukaryotes", "Peroxisome" and "Autophagy" (Fig S3, Table S4).

Another type is significantly up-regulated metabolite (D, L-typotophan) in the resistant soybean PI437654 but simultaneously dramatically down-regulated in the three susceptible soybean varieties. During the top 
150 most associated genes, 80 genes had positive correlations, while 70 genes had negative correlations (Fig. 4; Table S5). As for these associated genes, the most abundant GO terms in the biological processes category were "response to chitin", followed by "ethylene-activated signaling pathway" and "defense response" (Fig. 5; Table S6). Regarding the cellular component category, the most abundant G0 terms were "extracellular region", "apoplast" and "photosystem I" (Fig. 5; Table S6). Concerning the molecular function category, the most abundant GO terms were "transcription factor activity, sequence-specific DNA binding", "heme binding" and "hydroquinone:oxygen oxidoreductase activity" (Fig. 5; Table S6). Correspondingly, these associated genes were enriched in KEGG pathways, which involving the largest number of unigenes were "Plant hormone signal transduction", followed by "Phenylpropanoid biosynthesis", "MAPK signaling pathway - plant", "Starch and sucrose metabolism" and "Photosynthesis" (Fig S4, Table S7).

These results of the combination of metabolomic analyses and transcriptomics revealed potential associated genes involved in the most significantly up-regulated metabolites of the resistant soybean variety PI437654, and suggested their likely biological processes, cellular component, molecular function and involved pathways.

\section{Discussion}

A resistant soybean PI 437654 and three susceptible soybeans WM82, ZH13 and HF47 were used as the test materials to explore the changes of metabolites in soybean roots with and without SCN inoculation by LC/MS analyses in this study. Totally, 37 differential metabolites and 20 KEGG metabolic pathways were identified, which were divided into three categories including the metabolites/metabolic pathways overlapped among all the tested resistant and susceptible soybeans, specific in the susceptible soybeans and specific in the resistant soybeans (Table 1; Table 2). It was found that the same differential metabolites such as linolenic acid and L-arginine were probably involved in more than one KEGG metabolite pathways, while the differently differential metabolites such as nicotine and pipecolinic acid were likely involved in the identical tropane, piperidine and pyridine alkaloid biosynthesis pathway (Table 2). However, only 12 differential metabolites (32\%) were found to be involved in the predicted KEGG metabolite pathways.

In this study, some basal defense-related potential metabolites, such as linolenic acid and 16hydroxyhexadecanoic acid, were found in the resistant soybean (PI 437654) and the three susceptible soybeans (WM82, ZH13 and HF47), and our metabolomic analyses results were consistent with some previous reports. Linolenic acid is involved in the metabolic pathways including alpha-linolenic acid metabolism and biosynthesis of unsaturated fatty acids, and it was thought to be related to plant tolerance to low temperature, and the content of linolenic acid was proportional to the cold tolerance of wheat $[29,56,62]$. In addition, linolenic acid plays an important role in the defense response to pathogen infection and traumatic stress in Arabidopsis thaliana [7]. Unsaturated fatty acids are considered as a new generation of plant disease inducers. Amruthesh et al. (2005) investigated the role of unsaturated fatty acids in the resistance of pearl millet to downy mildew, and found that its stimulation induced 
strong plant resistance [2]. Clay et al. (2006) found that the host resistance was related to the higher content of unsaturated fatty acids in the nuclear membrane of resistant species on Gossypium barbadense [16].

The metabolites and metabolic pathways specific in the resistant soybean PI 437654 infected with SCN, mainly including L-arginine, phytosphingosine, $\mathrm{D}$-aspartic acid and nicotine, probably played pivotal roles on plant stress, tolerance or resistance based on some related research literature. L-arginine is the precursor of nitric oxide (NO), while NO is synthesized by L-arginine through NO synthase in the Larginine-NO pathway, which is widely considered as an important regulator of cell function and communication in physiological and pathophysiological states $[23,46,47]$. NO is a second messenger in the stress response, and is involved in plant responses to biotic and abiotic stresses $[18,66]$. L-arginine is linked and involved in many KEGG metabolic pathways such as arginine and proline metabolism, aminoacyl-tRNA biosynthesis and ABC transporters. Many studies indicated that there was a positive correlation between proline accumulation and plant stress tolerance [8]. Aminoacyl-tRNA synthase is an important component of protein synthesis and catalyzes the connection of amino acids with their homologous tRNA. They form a different set of enzymes, ensuring the fidelity of the transfer of genetic information from DNA to proteins [ 50,55$]$. ABC transporter is a member of the protein superfamily, it uses the energy from the hydrolysis of ATP to transport material over membranes [60]. Recently, ABC transporters attracted more attentions because the $A B C$ gene was associated with pesticide resistance [25]. Phytosphingosine (PHS), a member of the sphingosine family, shows fungicidal activity [57]. Castro et al. (2008) studied the effect of PHS on cells of the Neurospora crassa and found that PHS reduced the viability of fungal cells and induced fungal apoptosis [13]. D-aspartic acid participated in alanine, aspartate and glutamate metabolism, while glutamate dehydrogenase (GDH) is a central enzyme in glutamate metabolism [5]. The enhancement of drought resistance in wheat was related to ammonia assimilation and enhanced NADH-GDH activity under low osmotic stress and high osmotic stress [65]. Nicotine was widely used in killing many kinds of insects [28]. Vänninen at el. (2011) discovered that nicotine has a strong killing effect on the adults of miridae [58]. Imidacloprid is a new generation of nicotine pesticides with low toxicity, and nicotine and imidacloprid reduced aphid fecundity by inhibiting the reproduction of nymphs with certain concentrations [19]. Nicotine is involved in the biosynthesis of tropane, piperidine and pyridine alkaloid in metabolic pathways, and alkaloid was found to have obvious inhibitory effect on sunflower downy mildew fungus Plasmopara halstedii [51]. Thus, L-arginine, phytosphingosine, D-aspartic acid and nicotine may be involved in the resistance of soybean against SCN.

Differently, the differential metabolite 2-oxo-4-methylthiobutanoic acid, which was overlapped in all the three susceptible soybeans but absent in resistant soybean, was predicted to participate into three KEGG metabolic pathways, including cysteine and methionine metabolism, glucosinolate biosynthesis and 2oxocarboxylic acid metabolism. Moreover, 2-oxo-4-methylthiobutanoic acid was simultaneously downregulated in the three susceptible soybeans ( $\mathrm{ZH} 13$, WM82 and HF47) infected with SCN, which likely resulted in decreased biosynthesis of glucosinolate. It was reported that glucosinolate was a plant secondary metabolite and had a preventive effect against plant pathogens and soil-borne plant pests [10, 
37]. Additionally, the metabolic pathways of flavonoid biosynthesis, diterpenoid biosynthesis, which were reported to be involved in plant resistance to pathogens, were found to be enriched in one or two susceptible soybean varieties $[6,48]$. This type of differential metabolites and their related KEGG metabolite pathways might play potential roles in the manipulation of host innate immunity to establish and maintain the feeding sites for SCN development [26,54].

A local metabolite-calibrated database was used to identify potential differential metabolites in this study, which safeguarded the accuracy and precision [38,39], but also likely had some disadvantages to miss many important compounds due to its limited capacities. Nevertheless, in this study, the different metabolomic responses of the resistant and susceptible soybean varieties to SCN infection were thoroughly characterized, and some important and pivotal different metabolites and related KEGG metabolite pathways were identified, and their likely functions were predicted based on previous studies.

In this study, three key metabolites including $\mathrm{N}$-acetyltranexamic acid, nicotine, and D, L-typotophan, which were significantly up-regulated in the resistant soybean PI437654 infected by SCN, were identified and classified into two types. Combination of the metabolomic analyses with the transcriptomic expression profiling aided to predict potential associated genes involved in the most significantly upregulated metabolites of the resistant soybean variety PI437654, and suggest their likely biological processes, cellular component, molecular function and involved pathways.

\section{Conclusion}

In this study, the main metabolomic differences of the resistant and susceptible soybeans infected with SCN were characterized by using a broad-spectrum SCN-high resistant soybean PI 437654 versus three SCN-susceptible soybean varieties. Totally, 37 differential metabolites and 20 KEGG metabolic pathways were identified, which were divided into three categories including the metabolites/metabolic pathways overlapped among all the tested resistant and susceptible soybean, specific in susceptible or resistant soybeans. Twelve differential metabolites were found to be involved in the predicted KEGG metabolite pathways. Moreover, 14 differential metabolites such as significantly up-regulated nicotine and downregulated D-aspartic acid, and their involved KEGG pathways such as tropane, piperidine and pyridine alkaloid biosynthesis, alanine, aspartate and glutamate metabolism, sphingolipid metabolism and arginine biosynthesis were significantly changed and abundantly enriched specifically in the resistant soybean PI 437654, and likely played pivotal roles in defensing SCN. Moreover, combination of the metabolomic analyses with the transcriptomic expression profiling aided to predict potential associated genes involved in the most significantly up-regulated metabolites of the resistant soybean variety PI437654, and suggest their likely biological processes, cellular component, molecular function and involved pathways. To our best knowledge, our study was the first to investigate the different responses of resistant and susceptible soybeans infected with SCN by combining metabolomic analyses and transcriptomics. Our results not only found out the potential novel metabolites and associated genes in the resistant response of soybean to soybean cyst nematode, but also provided new insights into the interactions between soybean and soybean cyst nematode. 


\section{Methods}

\section{Soybean seedling preparation and SCN inoculation}

All the soybean seeds including PI 437654, Williams 82 (WM82), Zhonghuang 13 (ZH13) and Hefeng 47 (HF47), which were stored in the Institute of Plant Protection, Chinese Academy of Agricultural Sciences, were sterilized with $1.0 \%(\mathrm{w} / \mathrm{v}) \mathrm{NaClO}$ for $5 \mathrm{~min}$ [20], and the residual $\mathrm{NaClO}$ was washed with sterilized water. Then the seeds were placed between moist sterilized filter papers at $26^{\circ} \mathrm{C}$. About $48 \mathrm{~h}$, the germinated seeds were transplanted into the sandy soil (soil: sand $=3: 1(\mathrm{v} / \mathrm{v})$ ). The day and night temperature was set at $28{ }^{\circ} \mathrm{C} / 26^{\circ} \mathrm{C}$ with a photoperiod of $12 \mathrm{~h}$ [15].

The cysts of SCN race 4 were soaked in the $3 \mathrm{mM} \mathrm{ZnCl}_{2}$ solution and incubated in the dark at $25^{\circ} \mathrm{C}$ [52]. The hatched SCN J2s were collected with a glass tube, and the concentration of SCN J2s suspension was adjusted to $500 \mathrm{~J} 2 \mathrm{~s} /$ tube [41]. The 14-day-old soybean seedlings were inoculated with $1000 \mathrm{SCN}$ $\mathrm{J} 2 \mathrm{~s}$ / seedling. At 8 days post inoculation (dpi), the root samples of the resistant soybean PI437654 and the three susceptible soybean WM82, ZH13 and HF47, as well as their corresponding controls, were harvested. Each type of root sample was divided into two parts for the following metabolomic and transcriptomic analyses, respectively.

\section{Juveniles Staining And Cysts Counting}

Soybean root samples were collected at $8 \mathrm{dpi}$, and were stained by acid magenta staining as previously described [11], and the morphology were observed and the numbers of SCN juveniles within the root tissues were counted under a microscope (OLYMPUS-SZ2-ILST). The cysts were harvested from both the root tissues and root soil at $60 \mathrm{dpi}$, and their numbers were counted with a stereoscope (OLYMPUSBX53F).

\section{Lc/ms Metabolic Sequencing}

At $8 \mathrm{dpi}$, the root samples of the resistant soybean PI437654 and the three susceptible soybean WM82, $\mathrm{ZH} 13$ and HF47, as well as their corresponding controls, were harvested, which were named PI437654_SCN, PI437654_0, WM82_SCN, WM82_0, ZH13_SCN, ZH13_0, HF47_SCN and HF47_0, respectively. Each soybean root sample was ground into powder in liquid nitrogen. Equivalent $80 \mathrm{mg}$ root powder was dropped into a $1.5 \mathrm{~mL}$ centrifuge tube. Add $20 \mu \mathrm{L}$ internal standard $(0.3 \mathrm{mg} / \mathrm{mL} \mathrm{L-2-}$ chlorophenylalanine) and $1 \mathrm{~mL} 70 \%$ methanol (methanol :water $=7: 3$ (volume ratio)); Place at $-20{ }^{\circ} \mathrm{C}$ for 2 min to pre-cool, and grind with a grinding machine for $2 \min (60 \mathrm{~Hz})$; Then, ultrasonic extraction was placed at $4{ }^{\circ} \mathrm{C}$ for $30 \mathrm{~min}$; after standing at $-20^{\circ} \mathrm{C}$ for $20 \mathrm{~min}$, the samples were centrifuged for $15 \mathrm{~min}$ $\left(13,000 \mathrm{rpm}, 4^{\circ} \mathrm{C}\right)$, and $200 \mu \mathrm{L}$ supernatant was drawn with a syringe, each sample was repeated 3 times, and the same source supernatants were combined together. After filtering with a $0.22 \mu \mathrm{m}$ organic phase 
pinhole filter, it was transferred to an LC injection vial and stored at $-80^{\circ} \mathrm{C}$ for LC-MS analysis [63]. Each treatment had six repetitions.

\section{Metabolomic Analyses}

The metabolites were identified using a local metabolite-calibrated database [38, 39]. Multivariate statistical analysis, including principal component analysis (PCA) and partial least squares discriminant analysis (PLS-DA), was performed using SIMCA-P ${ }^{+} 14.0$ version software (Umetrics, Umeå, Sweden) to distinguish differences in metabolic profiles between pairwise groups. The Hotelling's T2 region, shown as an ellipse in score plots of the models, defines the $95 \%$ confidence interval of the modeled variation. In the t-test, a variable with $p$-value $<0.05$ was considered as a difference variable. Metabolites with the VIP (variable importance in the projection) $>1.0$ and $p$-value $<0.05$ were selected as differential metabolites. KEGG pathways were predicted by the differential metabolites with the $p$-value $<0.05$ [63].

\section{Transcriptomics Analyses}

For RNA extraction, the same six root samples were sampled and randomly divided into two groups for each soybean variety, representing two biological repeats. The mixed samples were named K45 and K31 (PI437654_SCN, PI437654_0), K46 and K33 (WM82_SCN, WM82_0), K47 and K35 (ZH13_SCN, ZH13_0), K48 and K37 (HF47_SCN, HF47_0), respectively. The total RNA isolation, RNA quality examination, library construction and RNA-Seq, sequence analyses, GO (Gene Ontology) and KEGG analyses were preformed as previously described $[14,36]$. Genes with an absolute value of log2 (fold change) $>0.5$ were defined as the differentially expressed genes (DEGs).

\section{Correlation Analysis Between Metabolome And Transcriptome Data}

Correlation Analyses were preformed as previously described [14]. Pearson correlation coefficients were calculated for metabolome and transcriptome data integration. The mean content of metabolites of different soybean varieties (the resistant soybean PI437654 and the three susceptible soybean WM82, $\mathrm{ZH13}$ and HF47) and the mean value of transcript abundance of DEGs together were calculated. The coefficients were calculated from log2 (fold change) of each metabolite and log2 (fold change) of each transcript. Correlations corresponding to a coefficient with R2 $>0.9$ were selected. The relationships between metabolome and transcriptome data were demonstrated by using Cytoscape (The Cytoscape Consortium, San Diego, CA, USA, version 2.8.2). Top 150 associated genes were focused on in this study.

\section{Declarations}


Thanks to Dr. Peng Huan from Institute of Plant Protection, Chinese Academy of Agricultural Sciences for the methodological suggestions for the revision of this article. Thanks to Dr.Vijai Bhadauria from China Agricultural University for critical read proof.

\section{Authors' contributions}

LAK and DLP conceived the study; XS and LAK wrote the manuscript. XS and QSC performed the experiments; XS and QSC conducted statistical analyses; SML revised the manuscript. All of the authors discussed the results and commented on the manuscript, and approved its submission for potential publication.

\section{Funding}

This project was supported by the Natural Science Foundation of China (31872924), the National Key R\&D Program of China (2018YFD0201000), and National Technology System of Soybean Industry (CARS-04-PS05). The funders had no role in study design, data collection and analysis, data interpretation, or in writing of the manuscript.

\section{Availability of data and materials}

All data generated during this study are included in this published article and its supplementary information files, and the raw data used or analysed were submitted to China National GeneBank (No.CNP0001271).

\section{Ethics approval and consent to participate}

Not applicable.

\section{Consent for publication}

Not applicable.

\section{Competing interests}

The authors declare that they have no competing interests.

\section{Author details}

${ }^{1}$ Institute of Plant Protection, Chinese Academy of Agricultural Sciences, Beijing 100193, China

${ }^{2}$ Zhengzhou Tobacco Research Institute of CNTC, Zhengzhou, Henan, China

${ }^{3}$ Soybean Research Institute, Heilongjiang Academy of Agricultural Sciences, 150086 Harbin, China

\section{References}


1. Afzal AJ, Natarajan A, Saini N, Iqbal MJ, Geisler M, Eishemy HA, Mungur R, Willmitzer L, Lightfoot DA. The nematode resistance allele at the Rhg 1 locus alters the proteome and primary metabolism of soybean roots. Plant Physiol. 2009;151:1264-80.

2. Amruthesh KN, Geetha NP, Jørgensen HJL, Neergaard ED, Shetty HS. Unsaturated fatty acids from zoospores of Sclerospora graminicola induce resistance in pearl millet. Eur J Plant Pathol. 2005;111:125-37.

3. Anand SC, Gallo KM, Baker IA, Hartwig EE. Soybean plant introductions with resistance to races 4 or 5 of soybean cyst nematode. Crop. Sci. 1988;28.

4. Anand SC, Wrather JA, Shumway CR. Soybean genotypes with resistance to race of soybean cyst nematode. Crop. Sci. 1985;25.

5. Andreas P. Glutamate dehydrogenase deficiency in cerebellar degener-ations: clinical, biochemical and molecular genetics aspects. Can J Neurol Sci. 1993;20:109-S16.

6. Arfaoui A. Effect of rhizobium isolates on isoflavonoid levels in chickpea plants infected with Fusarium oxysporum f.sp. ciceris. Phytopathol Mediterr. 2006;45:24-34.

7. Asanori Y, Takashi Y, Morifumi H, Hideharu S, Jeanluc M, Kensuke K, Shigemi S, Koh I. Disease resistance against Magnaporthe grisea is enhanced in transgenic rice with suppression of $\omega-3$ fatty acid desaturases. Plant Cell Physiol. 2007;48:1263-74.

8. Ashraf M, Foolad MR. Roles of glycine betaine and proline in improving plant abiotic stress resistance. Environ Exp Bot. 2007;59:206-16.

9. Borges RM, Rahil T, Santana DSJ, Andrade BT. Dereplication of plant phenolics using a massspectrometry database independent method. Phytochem Analysis. 2018;29:601-12.

10. Brown PD, Morra MJ. Control of soil-borne plant pests using glucosinolate-containing plants. Adv Agron. 1997;61:161-231.

11. Bybd DWJ, Kirkpatrick T, Barker KR. An improved technique for clearing and staining plant tissues for detection of nematodes. J Nematol. 1983;15:142-3.

12. Castro-Moretti FR, Gentzel I, Mackey A. Metabolomics as an emerging tool for the study of plantpathogen interactions. Metabolites. 2020;10.

13. Castro A, Lemos C, Falcao A, Glass NL, Videira A. Increased resistance of complex I mutants to phytosphingosine-induced programmed cell death. J Biol Chem. 2008;283:19314-21.

14. Chen Q, Li M, Wang C, Li Z, Xu J, Zheng Q, Liu P, Zhou H. Combining targeted metabolites analysis and transcriptomics to reveal chemical composition difference and underlying transcriptional regulation in Maca (Lepidium Meyenii Walp.) ecotypes. Genes (Basel). 2018;9.

15. Chen X, Li S, Zhao X, Zhu X, Duan Y. Modulation of (homo) glutathione metabolism and $\mathrm{H}_{2} \mathrm{O}_{2}$ accumulation during soybean cyst nematode infections in susceptible and resistant soybean cultivars. Int J Mol Sci. 2020;21:388.

16. Clay WF, Bartkowski EJ, Katterman FRH. Nuclear deoxyribonucleic acid metabolism and membrane fatty acid content related to chilling resistance in germinating cotton (Gossypium barbadense). 
Physiol Plantarum. 2006;38:171-5.

17. Cook DE, Lee TG, Guo X, Melito S, Wang K, Bayless AM, Wang J, Hughes TJ, Willis D. K. Copy number variation of multiple genes at $R h g 1$ mediates nematode resistance in soybean. Science. 2012;338:1206-9.

18. Delledonne M, Xia Y, Dixon RA. Nitric oxide functions as a signal in plant disease resistance. Nature. 1998;394:585-8.

19. Devine GJ, Harling ZK, Scarr AW, Devonshire AL. Lethal and sublethal effects of imidacloprid on nicotine-tolerant Myzus nicotianae and Myzus persicae. Pest Manag Sci. 1996;48:57-62.

20. Douillet P. Disinfection of rotifer cysts leading to bacteria-free populations. J Exp Mar Biol Ecol. 1998;224:183-92.

21. Duan Y, Zheng Y, Chen L, Zhou X, Wang Y, Sun J. Effects of abiotic environmental factors on soybean cyst nematode. Agr Sci China. 2009;8:63-71.

22. Eloh K, Sasanelli N, Maxia A, Caboni P. Untargeted metabolomics of tomato plants after root-knot nematode infestation. J Agr Food Chem. 2016;64:5963-8.

23. Epstein FH. The L-arginine-nitric oxide pathway. New Engl J Med. 1993;329:2002.

24. Han Y, Zhao X, Cao G, Wang Y, Li Y, Liu D, Teng W, Zhang Z, Li D, Qiu L. Genetic characteristics of soybean resistance to $H G$ type 0 and $H G$ type 1.2.3.5.7 of the cyst nematode analyzed by genomewide association mapping. BMC Genomics. 2015;16:598.

25. Hans $M$. ABC transporters and their role in protecting insects from pesticides and their metabolites. Adv Insect Physiol. 2014;46:1-72.

26. Hewezi T, Baum TJ. Manipulation of plant cells by cyst and root-knot nematode effectors. Mol Plant Microbe in. 2013;26:9-16.

27. Hu Y, You J, Li C, Williamson VM, Wang C. Ethylene response pathway modulates attractiveness of plant roots to soybean cyst nematode Heterodera glycines. Sci Rep. 2017;7:41282.

28. Huang C, Ren J, Liang Y. Studies on isolation of nicotine from tobacco and its effects on killing insects. Acta. Agric. Univ. Jiangxiensis. 2001.

29. John JB, Christiansen MN. Inhibition of linolenic acid synthesis and modification of chilling resistance in cotton seedlings. Plant Physiol. 1976;57:257-9.

30. Jones MGK. The development and function of plant cells modified by endoparasitic nematodes. Elsevier Inc. 1981.

31. Kandoth PK, Ithal N, Recknor J, Maier T, Nettleton D, Baum TJ, Mitchum MG. The soybean Rhg1 locus for resistance to the soybean cyst nematode Heterodera glycines regulates the expression of a large number of stress and defense-related genes in degenerating feeding cells. Plant Physiol. 2011;155:1960-75.

32. Kang W, Zhu X, Wang Y, Chen L, Duan Y. Transcriptomic and metabolomic analyses reveal that bacteria promote plant defense during infection of soybean cyst nematode in soybean. BMC Plant Biol. 2018;18:86. 
33. Kantor M, Levi A, Thies J, Guner N, Parnham S. NMR analysis reveals a wealth of metabolites in rootknot nematode resistant roots of citrullus amarus watermelon plants. J Nematol. 2018;50:303-16.

34. Klink VP, Overall CC, Alkharouf NW, Macdonald MH, Matthews BF. Laser capture microdissection (LCM) and comparative microarray expression analysis of syncytial cells isolated from incompatible and compatible soybean (Glycine max) roots infected by the soybean cyst nematode (Heterodera glycines). Planta. 2007;226:1389-409.

35. Klink VP, Overall CC, Alkharouf NW, Macdonald MH, Matthews BF. A time-course comparative microarray analysis of an incompatible and compatible response by Glycine max (soybean) to Heterodera glycines (soybean cyst nematode) infection. Planta. 2007;226:1423-47.

36. Kong L, Wu D, Huang W, Peng H, Wang G, Cui J, Liu S, Li Z, Yang J, Peng D. Large-scale identification of wheat genes resistant to cereal cyst nematode Heterodera avenae using comparative transcriptomic analysis. BMC genomics. 2015;16:1-18.

37. Koroleva OA, Gibson TM, Cramer R, Stain C. Glucosinolate-accumulating S-cells in Arabidopsis leaves and flower stalks undergo programmed cell death at early stages of differentiation. Plant J. 2010;64:456-69.

38. Liu P, Luo J, Zheng Q, Chen Q, Zhai N, Xu S, Xu Y, Jin L, Xu G, Lu X. Integrating transcriptome and metabolome reveals molecular networks involved in genetic and environmental variation in tobacco. DNA. Res. 2020;27.

39. Liu P, Zhou H, Zheng Q, Lu P, Yu Y, Cao P, Chen W, Chen Q. An automatic UPLC-HRMS data analysis platform for plant metabolomics. Plant Biotechnol J. 2019;17:2038-40.

40. Liu S, Kandoth PK, Warren SD, Yeckel G, Heinz R, Yang AJ, Jamai C, Eimellouki A, Juvale T. PS. A soybean cyst nematode resistance gene points to a new mechanism of plant resistance to pathogens. Nature. 2012;492:256-60.

41. Lopeznicora HD, Craig JP, Gao X, Lambert KN, Niblack TL. Evaluation of cultivar resistance to soybean cyst nematode with a quantitative polymerase chain reaction assay. Plant Dis. 2012;96:1556-63.

42. Manchester M, Anand A. Metabolomics:strategies to define the role of metabolism in virus infection and pathogenesis. Adv Virus Res. 2017;98:57-81.

43. Masonbrink R, Maier TR, Muppirala U, Seetharam AS, Lord E, Juvale PS, Schmutz J, Johnson NT, Korkin D, Mitchum MG. The genome of the soybean cyst nematode (Heterodera glycines) reveals complex patterns of duplications involved in the evolution of parasitism genes. BMC Genomics. 2019;20:119.

44. Matthews KBF, Klink VP. Emerging approaches to broaden resistance of soybean to soybean cyst nematode as supported by gene expression studies. Plant Physiol. 2009;151:1017-22.

45. Mazarei M, Liu W, Alahmad H, Arelli PR, Pantalone VR Jr. CNS. Gene expression profiling of resistant and susceptible soybean lines infected with soybean cyst nematode. Theor Appl Genet. 2011;123:1193-206.

46. Moncada S, Higgs A. The L-arginine-nitric oxide pathway. New Engl J Med. 1993;329:2002-12. 
47. Moncada S, Palmer RMJ, Higgs EA. Biosynthesis of nitric oxide from L-arginine: a pathway for the regulation of cell function and communication. Biochem Pharmacol. 1989;38:1709-15.

48. Moustafa EM. Leaf curl virus and the flavonoid content of resistant and susceptible strains of cotton. Nature. 1961;191:415.

49. Niblack TL, Lambert KN, Tylka GL. A model plant pathogen from the kingdom Animalia: Heterodera glycines, the soybean cyst nematode. Annu Rev Phytopathol. 2006;44:283-303.

50. Odonoghue $P$, Lutheyschulten $Z$. On the evolution of structure in aminoacyl-tRNA synthetases. Microbiol Mol Biol Rev. 2003;67:550-73.

51. Oros G. Ujváry, István. Botanical fungicides: natural and semisynthetic ceveratrum alkaloids. Pest Manag Sci. 1999;55:253-64.

52. Peng H, Cui J, Long H, Huang W, Kong L, Liu S, He W, Hu X, Peng D, John J. Novel pectate lyase genes of Heterodera glycines play key roles in the early stage of parasitism. PLoS One. 2016;11:e0149959.

53. Rachel C, Danyel J, Jos K, Jan BJJ. Transcriptomic and metabolomic data integration. Brief Bioinform. 2015;5:891-901.

54. Siddique S, Grundler FM. Parasitic nematodes manipulate plant development to establish feeding sites. Curr Opin Microbiol. 2018;46:102-8.

55. Szymański M, Deniziak M, Barciszewski J. The new aspects of aminoacyl-tRNA synthetases. Acta Biochim Pol. 2000;47:821-34.

56. Torresfranklin ML, Repellin A, Huynh VB, Arcylameta A, Zuilyfodil Y, Phamthi AT. Omega-3 fatty acid desaturase (FAD3, FAD7, FAD8) gene expression and linolenic acid content in cowpea leaves submitted to drought and after rehydration. Environ Exp Bot. 2009;65:162-9.

57. Valentijnbenz M. Phytosphingosine kills Candida albicans by disrupting its cell membrane. Biol Chem. 2010;391:65-71.

58. Vänninen I, Rönnqvist M, Dahlqvist M, Forsström J. As an invasive pest in Finnish tomato crops: attempt to eradicate the bugs with nicotine-based programmes. lobc/wprs Bulletin. 2011.

59. Vuong TD, Sonah H, Meinhardt CG, Deshmukh R, Kadam S, Nelson RL, Shannon JG, Nguyen HT. Genetic architecture of cyst nematode resistance revealed by genome-wide association study in soybean. BMC Genom. 2015;16:593.

60. Waard MAD. Significance of ABC transporters in fungicide sensitivity and resistance. J Pestic Sci. 1997;51:271-5.

61. Wang Z, Tian Z. Genomics progress will facilitate molecular breeding in soybean. Sci China Life Sci. 2015;58:813-5.

62. Willemot $C$, Pelletier $L$. Influence of light and temperature on the linolenic acid content and frost resistance of winter wheat. Can. J. Plant. Sci. 1980.

63. Zeng C, Lin H, Liu Z, Liu Z. Metabolomics analysis of Camellia sinensis with respect to harvesting time. Food Res Int. 2020;128:108814. 
64. Zhang H, Li C, Davis EL, Wang J, Griffin JD, Kofsky J, Song B. Genome-wide association study of resistance to soybean cyst nematode (Heterodera glycines) HG Type 2.5.7 in wild soybean (Glycine soja). Front Plant Sci. 2016;7:1214.

65. Zhang HN, Wang ZQ, Cui GJ, Lin TB. Difference in seedlings ammonium assimilation of wheat cultivars with different drought resistance under osmotic stress. China J Appl Ecol. 2009;20:240610.

66. Zhou B, Guo Z, Xing J, Huang B. Nitric oxide is involved in abscisic acid-induced antioxidant activities in Stylosanthes guianensis. J Exp Bot. 2005;56:3223-8.

\section{Figures}

a

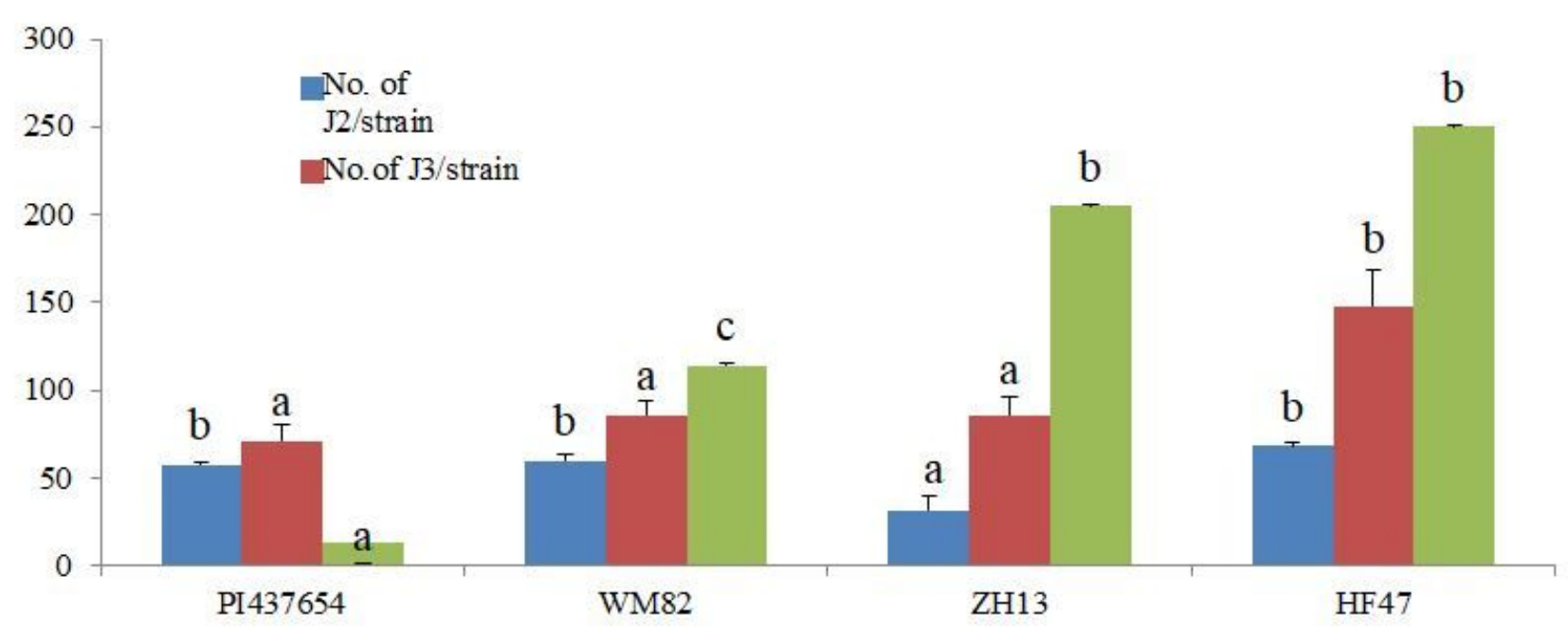

$\mathrm{b}$

PI437654

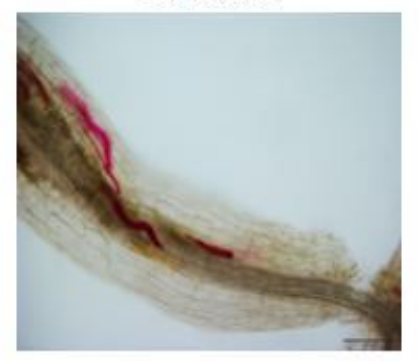

WM82

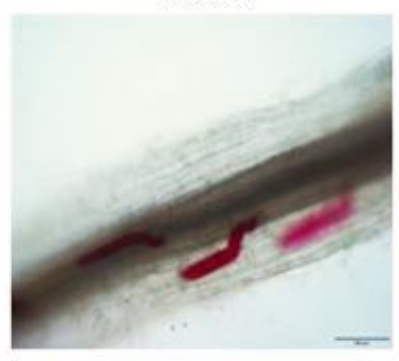

$\mathrm{ZH} 13$

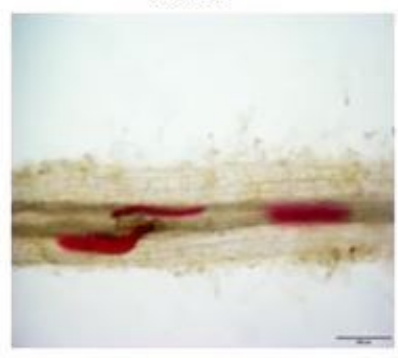

HF47

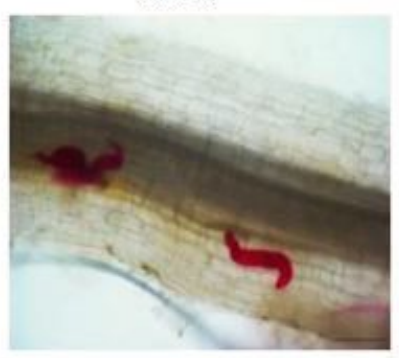

\section{Figure 1}

Assessment of the resistance of soybean lines to SCN. (a) The average numbers of J2s, J3s and cysts in the roots of the resistant soybean PI 437654 and three susceptible soybeans Williams 82 (WM82), Zhonghuang 13 (ZH13) and Hefeng 47 (HF47). (b) Morphological development of SCN in the roots of the resistant soybean PI 437654 and three susceptible soybeans WM82, ZH13 and HF47 at 8 dpi. Bar=200 $\mu \mathrm{m}$. 
a

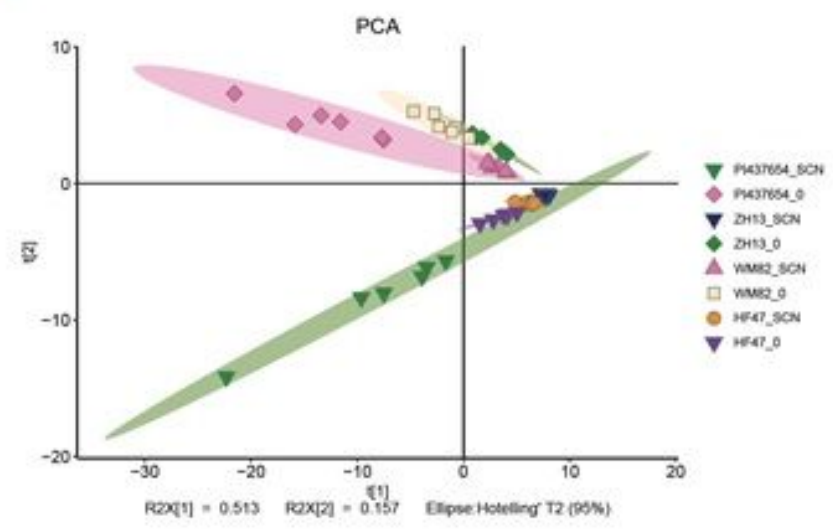

c

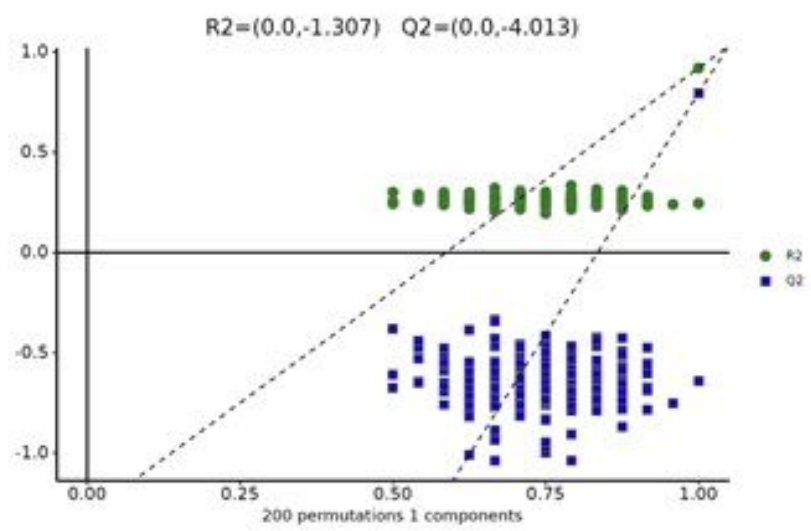

b

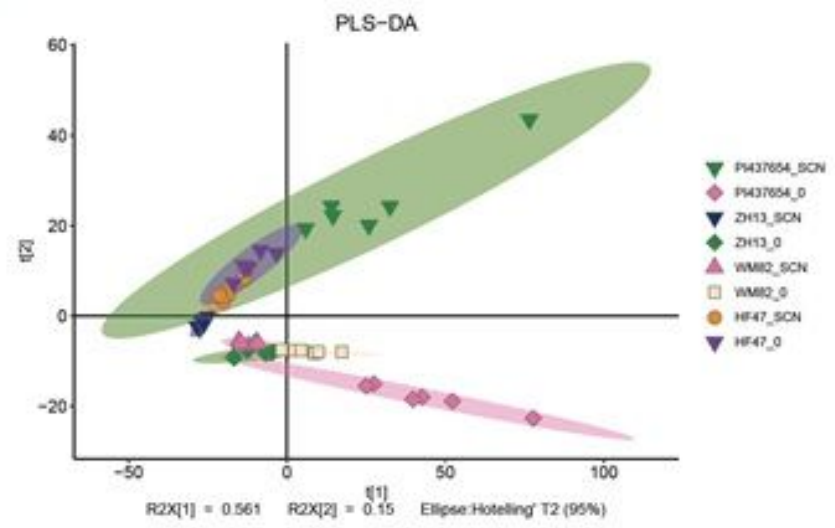

\section{Figure 2}

Principal Component Analysis (PCA), partial least-squares-discriminant analysis (PLS-DA) analysis and permutation tests of soybean metabolite profiling data. (a) PCA analysis, scores plot of principal components analysis of the resistant PI 437654 and three susceptible soybeans Williams 82 (WM82), Zhonghuang 13 (ZH13) and Hefeng 47 (HF47) infected with SCN. (b) PLS-DA score plots, based on metabolite profiling data of the resistant PI 437654 and three susceptible soybeans Williams 82 (WM82), Zhonghuang 13 (ZH13) and Hefeng 47 (HF47) infected with SCN. (c) Permutation tests of PLS-DA models, the permutation tests were carried out with 200 random permutations. Each point represents a metabolite profile of a biological replicate. 

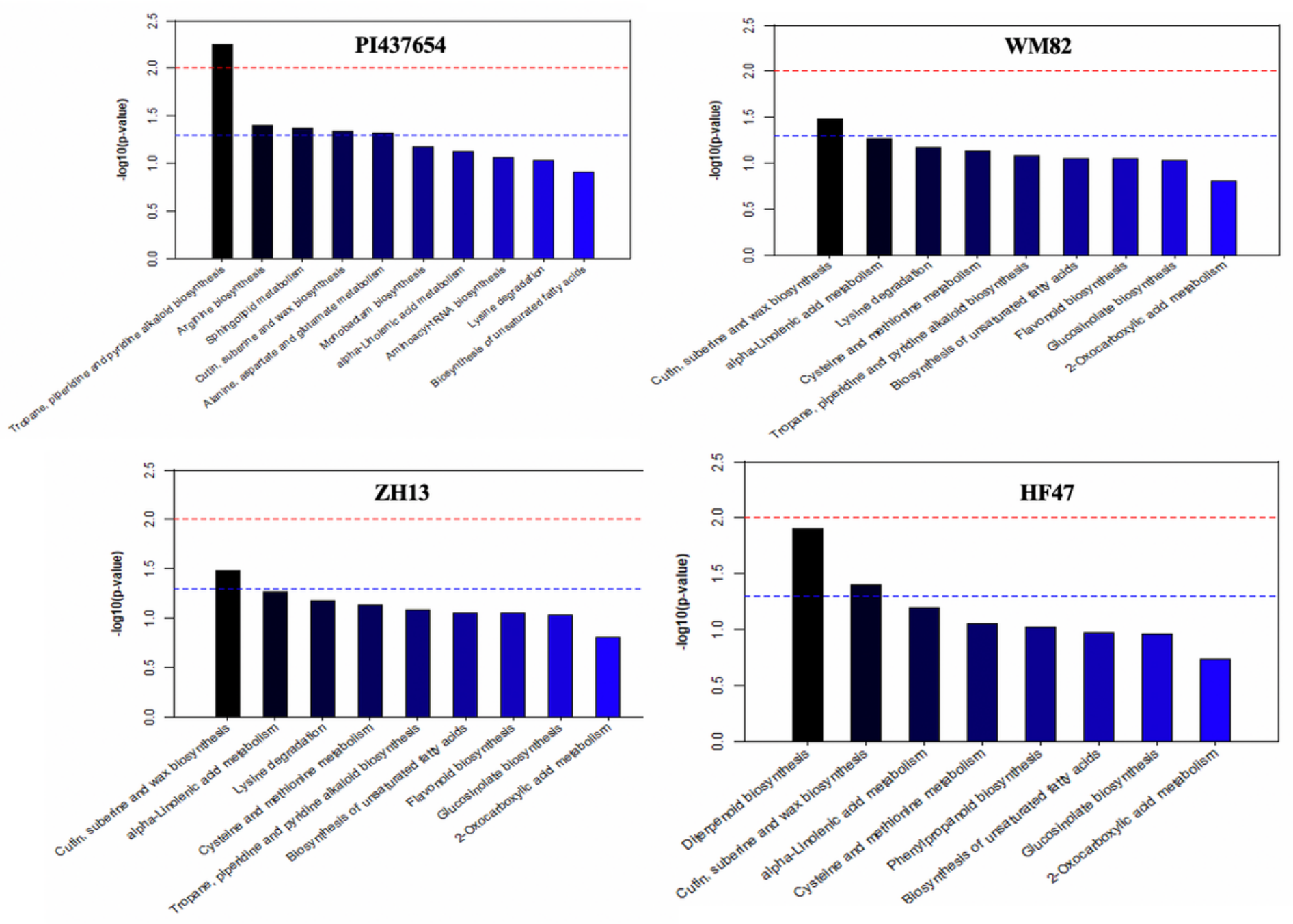

Figure 3

Metabolic pathways analyses of differential metabolites in the resistant PI 437654 and three susceptible soybeans Williams 82 (WM82), Zhonghuang 13 (ZH13) and Hefeng 47 (HF47). The red and blue dot lines denote the significance with $p<0.01 p<0.05$, respectively. The metabolic pathways with the top of the bar higher than the blue or the red dot lines were significantly changed. 


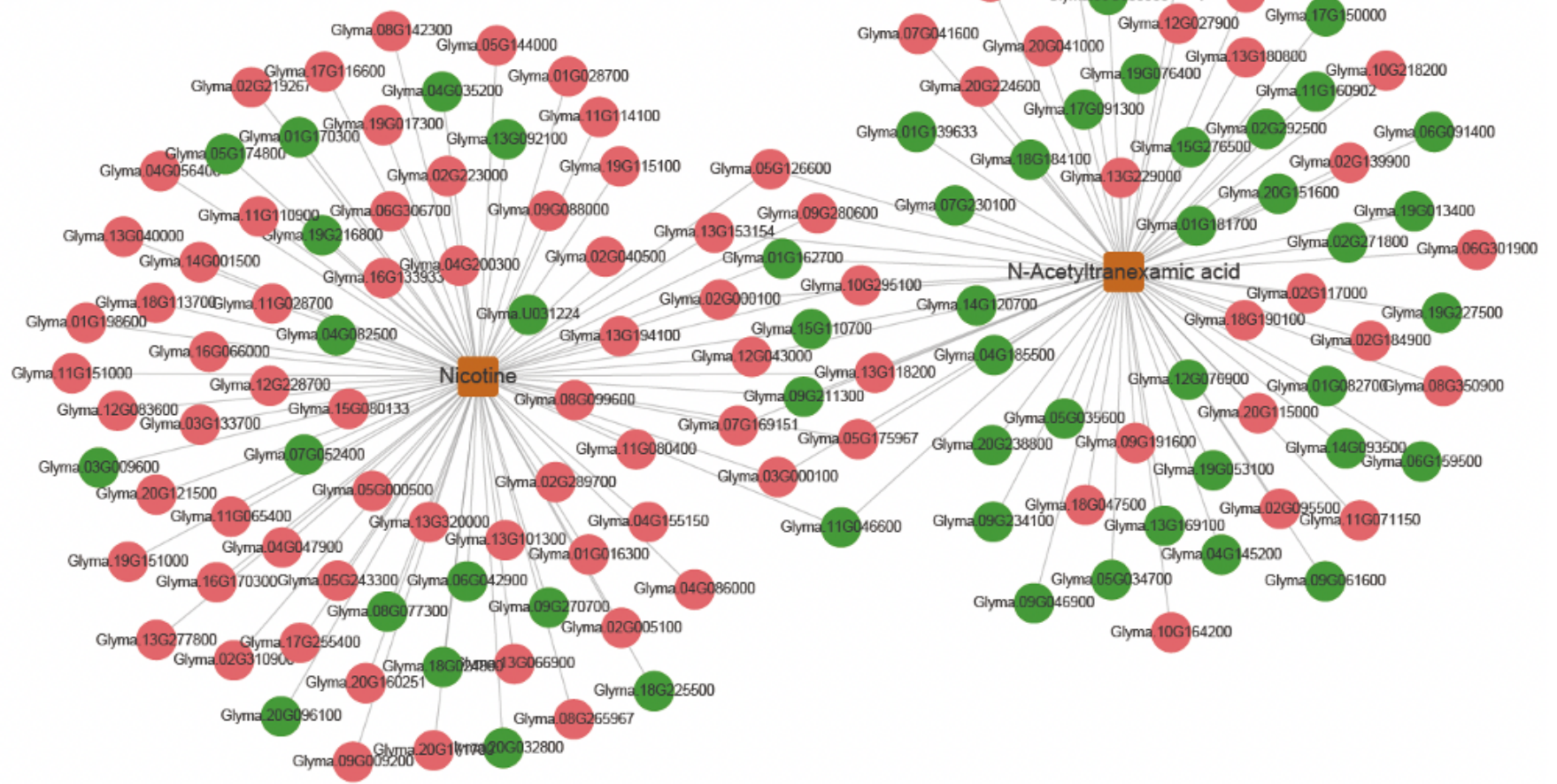

\section{B}

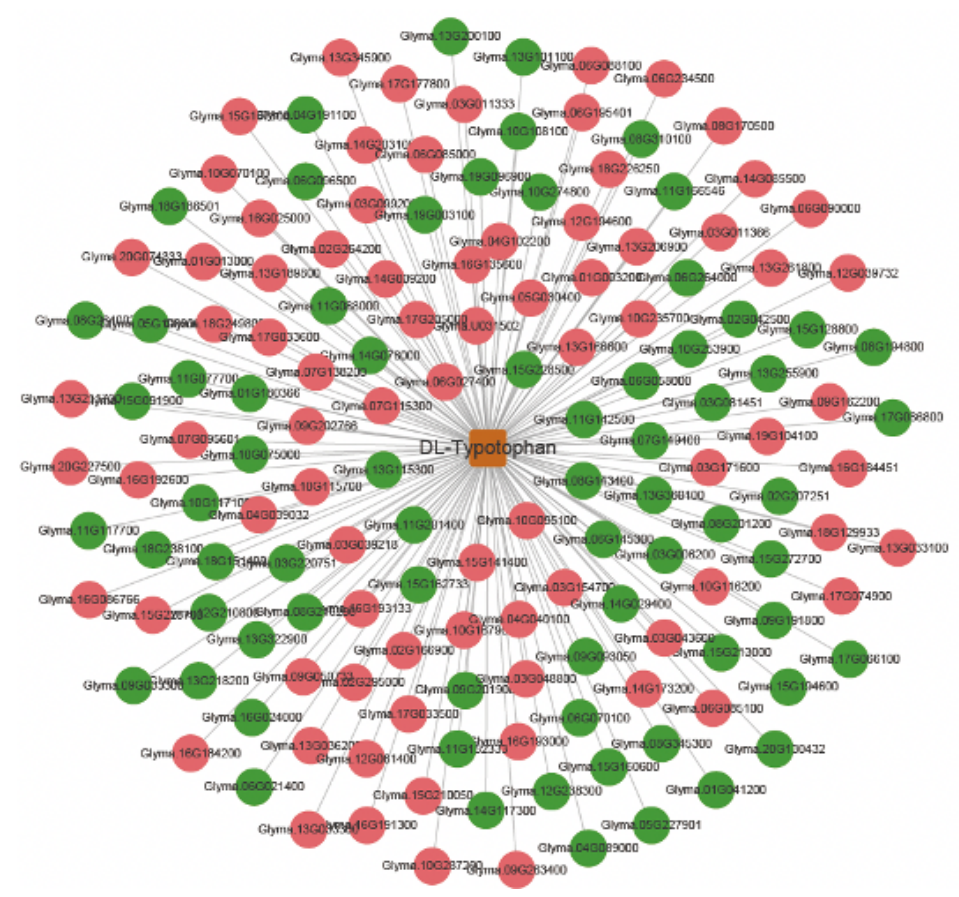

\section{Figure 4}

Connection network between the resistant soybean PI437654 - specific significantly up-regulated metabolites (a) and the overlapping metabolite with significantly up-regulated but simultaneously downregulated profiles. (b) and their associated regulatory genes, respectively. Red lines represent positive correlations and grey lines represent negative correlations. 

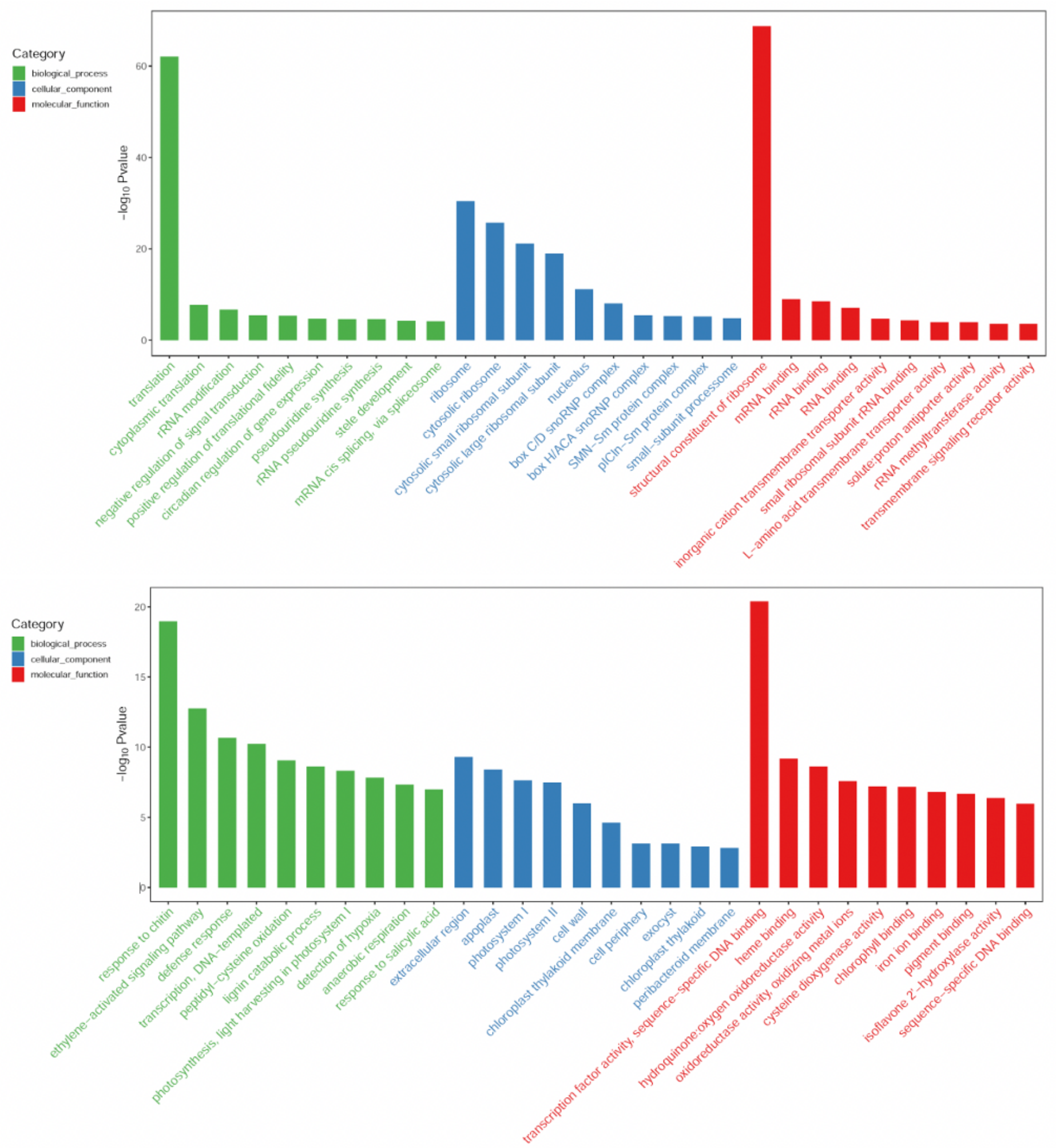

\section{Figure 5}

GO analyses of the genes associated with the resistant soybean PI437654 -specific significantly upregulated metabolites (upper) and the overlapping metabolites with significantly up-regulated but simultaneously down-regulated profiles (bottom), respectively. GO analyses of the genes associated with the resistant soybean PI437654 - specific significantly up-regulated metabolites (upper) and the 
overlapping metabolites with significantly up-regulated but simultaneously down-regulated profiles (bottom), respectively.

\section{Supplementary Files}

This is a list of supplementary files associated with this preprint. Click to download.

- TableS7.xIsx

- TableS6.xIsx

- TableS5.xIsx

- TableS4.xlsx

- TableS3.xlsx

- Tables2.xlsx

- Tables1.docx

- FigureS4.pptx

- FigureS3.pptx

- FigureS2.pptx

- FigureS1.pptx 